\title{
Influencia del estado de conducción, tipo de vehículo y condiciones de viaje en las variables cinemáticas de las rutas en autobuses urbanos. Caso de estudio Ibarra-Ecuador
}

\author{
(Influence of the Driving Cycle, Type of Vehicle and Travel \\ Conditions in the Cinematic Variables of the Routes \\ in Urban Buses. Case Study Ibarra-Ecuador)
}

\author{
Fredy Rosero', Zamir Mera², Ramiro Rosero ${ }^{3}$, Fausto Tapia ${ }^{4}$, Rommel Imbaquingo5 ${ }^{\text {, Jordán Ramirez }}{ }^{6}$
}

\begin{abstract}
Resumen
Las ciudades de Latinoamérica tienen problemas de contaminación y tráfico por la falta de investigación y planificación del sector de transporte. En Ecuador, las características de las flotas de transporte y su cinemática en la ciudad no se han determinado. El presente estudio analiza detalladamente las características cinemáticas de cada ruta de transporte público de la ciudad de Ibarra-Ecuador mediante el uso de equipos de georreferenciación GPS para cada segundo de recorrido. Se monitoreó 22 rutas de autobuses urbanos, evaluando 186 autobuses en 832 viajes, en los que se determinó que la velocidad promedio, la aceleración y desaceleración dependen, principalmente, de tres factores: ruta, sector de la ciudad y modelo de vehículo. Otros factores como operadora, edad de conductor, tiempos de viaje y la franja horaria no presentaron mayor significancia en la influencia a las variables cinemáticas en estudio, por lo que no se consideran dentro del estudio de investigación.
\end{abstract}

\section{Palabras clave}

Cinemática del automóvil, autobuses urbanos, congestión vehicular, estados de conducción, ciclos de conducción.

\begin{abstract}
Latin American cities have pollution and traffic problems due to the lack of research and planning in the transport sector. In Ecuador, the characteristics of the transport fleets and their kinematics in the city have not been determined. This study analyzes in detail the kinematic characteristics of each public transport route in the city of Ibarra-Ecuador using GPS georeferencing equipment for each second of travel. 22 urban bus routes were monitored evaluating 186 buses in 832 trips, where it was determined that the average speed, acceleration and deceleration depend mainly on three factors: route, city sector and vehicle model. Other factors such as operator, age of driver, travel times and time slot did not show greater significance in influencing the kinematic variables under study, so it is not considered within the research study.
\end{abstract}

\section{Keywords}

Vehicle kinematics, urban buses, vehicle congestion, driving styles, driving cycles. 


\section{Introducción}

Ante el incremento en la demanda del transporte y la movilidad urbana ha sido necesario analizar y resolver las problemáticas que estas traen consigo. Para ello, se han desarrollado modelos de cinemática vehicular para cumplir con los datos y las limitaciones experimentales, así como para abordar problemas particulares como el consumo de combustible y las emisiones vehiculares. Sin embargo, muchos de estos ciclos tienen limitaciones, por ejemplo, la propuesta de la Comisión Económica para Europa (Davari et al., 2017). En América latina, existen cambios drásticos, hoy en día el aumento en el tráfico urbano y extraurbano no es limitado. Por el contrario, se ha convertido en unos de los más grandes problemas que enfrentan las ciudades en crecimiento. El desarrollo de ciclos de conducción es crítico, ya que predecir éstos, basados en las condiciones de la ruta, muchas veces se ven afectados por varios factores inciertos que tienen un gran impacto en la investigación y tienen una relación directa con el uso de la velocidad (Huang et al., 2017).

El analizar la cinemática vehicular también ha conllevado a estudiar otros parámetros como, por ejemplo, lo relacionado con la contaminación. En el Ecuador, el sector transportista representa un consumo de combustibles de alrededor del $77 \%$, equivalente a 57 millones de barriles de petróleo, aproximadamente, datos que fueron emitidos por el Ministerio Coordinador de Sectores Estratégicos en el 2013 (Sierra, 2016). Pero la cantidad de contaminación generada por este sector aún no tiene una estimación real ya que muchas veces la calidad del combustible, la situación geográfica y el mal uso en la cinemática vehicular, debido a las paradas, arranques y tiempo de espera generados por los hábitos culturales de conductores y pasajeros, indica que difícilmente se tendrá un rendimiento específico para una zona determinada en los sistemas de transporte.

Todos estos tipos de conducción relacionados con los buses de transporte urbano pueden ser obtenidos de diferente manera, siendo el más eficiente el realizado on-board, mediante instrumentos adecuados que detectan las condiciones de manejo reales.

Esta técnica fue y es aplicada en la ciudad de Chennai en la India, logrando recopilar datos de velocidad y tiempo mediante dispositivos on-board GPS y, de este modo, se obtienen los ciclos de conducción para posteriormente calcular las fracciones de aceleración, desaceleración, velocidad crucero, desplazamiento y ralentí (Kancharla y Ramadurai, 2018).

Otra técnica es el análisis discriminante lineal basado en la comparación de los parámetros del ciclo de conducción y los datos de conducción del mundo real, esta fue aplicada en el desarrollo de ciclos de conducción en Tianjin. La singularidad de esta metodología se debe a que también se analiza en comparación con metodología tradicional en el desarrollo de ciclos, convirtiendose asi en una investigación que podría ofrecer una nueva metodología para la construcción de ciclos de conducción y referencia para investigaciones relacionadas (Jing et al., 2017).

Estudios también afirman que los factores medioambientales y financieros obligan a desarrollar vehículos limpios, eficientes y sostenibles, siendo la transición a la electrificación e hibridación una posible solución a estos problemas. Sin embargo, los ciclos de conducción también son soluciones, ya que representan las condiciones reales de conducción para las pruebas de emisiones de vehículos y estimación, por lo tanto, crear un ciclo de conducción práctico para vehículos con parámetros característicos como velocidad, distancia, tiempo y aceleración se convirte en algo muy importante, puesto que implica determinar la ruta y luego analizar los datos utilizando técnicas de muestreo estratificado (Kaymaz et al., 2019). 
Otros estudios, como por ejemplo el modelo de perfil de velocidad 'sintético' basado en el límite de velocidad de la ruta y los límites de aceleración del autobús, indican que los perfiles de velocidad representativos pueden predecirse por las regulaciones de ruta y los límites cinemáticos del vehículo (Minett et al., 2011).

Así también, en investigaciones previas realizadas se evidencia la obtención de datos de velocidad instantánea versus tiempo, así como datos de georeferenciación GPS que fueron procesados y analizados para desarrollar el ciclo de conducción típico en Hanoi, permitiendo la eficiencia del tránsito de autobuses y la dismunición de factores de emisiónes de contaminantes atmosféricos como el CO, CO2, VOC, NOx, SO2 y las PM10 basado en las normas de emisiones internacionales en vehículos (Nguyen et al., 2021).

Por lo tanto, de acuerdo a lo mencionado, se ha llevado a cabo este extenso estudio elaborando una base de datos con las características cinemáticas de cada ruta de transporte público de la ciudad de Ibarra. Se calculó el porcentaje de permanencia promedio de los autobuses en cada estado de marcha (aceleración, desaceleración, ralentí y velocidad crucero) y su relación con los tiempos de viaje y niveles de consumo de combustible. Para el presente estudio, el análisis estadístico se realizó debido a la gran cantidad de datos obtenidos como base de otras investigaciones, entre las cuales se logró realizar el mapa de zonificación de la ciudad de Ibarra, depurar las rutas, generar una tasa de ocupación por ruta, elaborar un análisis de edad promedio, tanto para vehículos como para conductores, y relacionarlo con el consumo de combustible.

\section{Metodología}

\subsection{Caracterización de los buses urbanos de lbarra}

En la ciudad de Ibarra operan dos cooperativas de buses de transporte público: la Cooperativa '28 de Septiembre' y la Cooperativa 'San Miguel de Ibarra'. Se obtuvo información de los vehículos como: marca, modelo y año de fabricación; datos que fueron facilitados por la Empresa Pública de Movilidad (MOVIDELNOR-EP). De la información recopilada se indica un total de 287 vehículos, 160 corresponden a la Cooperativa '28 de Septiembre' y 127 a la Cooperativa 'San Miguel de Ibarra' (Rosero et al., 2017).

Dentro de la caracterización de la flota, y de acuerdo con el modelo de fabricación, se identificaron las marcas que tienen mayor influencia dentro del parque automotor, siendo: Chevrolet Isuzu FTR 32M, Chevrolet Isuzu CHR 7.2 e Hino y Volkswagen 17210. Además, el promedio de año de fabricación de los autobuses es del 2002, por lo tanto, el bus promedio no cumple la Norma de emisiones de gases Euro V y Euro VI (Jääskeläinen y Khair, 2020)

\subsection{Caracterización de las rutas de Ibarra}

Para la caracterización de las rutas se realizó un inventario de las existentes para las flotas de autobuses de la ciudad de Ibarra. Actualmente, se cuenta con 22 rutas en funcionamiento, 13 rutas de la Cooperativa '28 de Septiembre' y 9 rutas de la Cooperativa 'San Miguel de Ibarra'. Además, se obtienen otros datos como: recorridos, frecuencias, horarios y tiempos de viaje y algunos datos que se muestran en la Tabla 1. 


\subsection{Selección de equipos GPS para monitoreo}

El levantamiento de los datos en ruta referentes a velocidad y tiempo para la obtención de estados de conducción para el cálculo de las fracciones de aceleración, desaceleración, velocidad crucero, desplazamiento y ralentí se registraron mediante un dispositivo on-board GPS, basándose en la metodología utilizada por Kancharla y Ramadurai (2018).

Tabla 1. Caracterización de las rutas de Ibarra

\begin{tabular}{|c|c|c|c|}
\hline Operadora & Rutas & $\begin{array}{c}\text { Distancia } \\
\quad(\mathbf{k m})\end{array}$ & $\begin{array}{c}\text { Tiempo } \\
\text { (min) }\end{array}$ \\
\hline \multirow{13}{*}{28 de Septiembre } & Aloburo & 20.38 & 70 \\
\hline & Arcángel - San Cristóbal & 35.26 & 120 \\
\hline & Azaya - La Campiña & 19.23 & 100 \\
\hline & Caranqui - Aduana & 26.75 & 110 \\
\hline & El Carmen - Bellavista & 23.82 & 75 \\
\hline & Católica - Alpachaca & 17.09 & 90 \\
\hline & Chorlaví - La Victoria & 33.71 & 130 \\
\hline & Milagro - Yahuarcocha & 34.84 & 125 \\
\hline & Naranjito & 21.63 & 100 \\
\hline & Las Palmas - Los Ceibos & 19.35 & 93 \\
\hline & Pugacho - Santa Teresita & 19.04 & 98 \\
\hline & Santa Rosa - Los Ceibos & 22.89 & 110 \\
\hline & Tanguarín - Aduana & 36.41 & 145 \\
\hline \multirow{9}{*}{$\begin{array}{c}\text { San Miguel } \\
\text { de Ibarra }\end{array}$} & Caranqui - Universidades & 20.59 & 75 \\
\hline & Chugchupungo - Florida & 24.27 & 110 \\
\hline & Colinas del Sur - Aduana & 25.85 & 100 \\
\hline & Ejido de Caranqui - Miravalle & 28.45 & 120 \\
\hline & La Esperanza - Hospital Seguro & 24.04 & 115 \\
\hline & 19 de Enero - Odilas & 29.08 & 130 \\
\hline & Santa Isabel - H. Familiares & 39.22 & 150 \\
\hline & Santa Lucía - La Victoria & 23.99 & 105 \\
\hline & Santo Domingo - Universidades & 30.27 & 120 \\
\hline
\end{tabular}

Se seleccionó un registrador de datos GL-770 Profesional GNSS Data Logger (Soporte de GPS y GLONASS) conformado por un receptor GPS. Este dispositivo incluye una retroalimentación audible y un sensor de movimiento que genera la máxima duración de la batería. Los datos se pueden obtener con una frecuencia de 1 a $5 \mathrm{~Hz}$, en un rango máximo de 35 horas de funcionamiento. Las pruebas piloto se realizaron para verificar el cumplimiento de las especificaciones técnicas y mejorar las destrezas en la operación de los mismos. 


\subsection{Levantamiento de datos de las rutas}

El levantamiento de datos se realizó en temporadas en las que las actividades educativas, laborales, administrativas, sociales y comerciales eran normales, lo que garantizó tener datos representativos en función de las condiciones cotidianas del tráfico de la ciudad. Se monitorearon todas las rutas de Ibarra durante todo el día en tres condiciones diferentes: semana (lunesviernes), sábado y domingo.

Cada condición es diferente, por lo tanto, conlleva que existan cambios de horarios, frecuencias, rutas y otros factores que afectan el estado de conducción. Logrando registrar 3600 datos por hora y por equipo.

En total, entre semana se monitorearon 92 buses, de los cuales se obtuvieron 472 viajes y 3130908 registros. Para los sábados se monitorearon 39 buses y se obtienen 195 viajes y 1274812 registros; mientras que los domingos se monitorearon 37 buses, dando como resultado 165 viajes y 1108146 registros. Logrando un tiempo total de grabación de 1532 horas, aproximadamente.

\subsection{Definición de variables y parámetros de estudio}

Para la determinación de las variables y los parámetros de estudio se toma en cuenta la cinemática del vehículo. Entre las variables y parámetros referenciales tenemos: 1) ubicación (m); 2) tiempo (s); 3) distancia recorrida en cada viaje (m); 4) número de autobuses y 5) número de días de estudio.

A partir de estos parámetros se determinaron las diferentes variables como son:1) velocidad; 2) velocidad instantánea; 3) velocidad promedio; 4) aceleración instantánea; 5) aceleración y desaceleración promedio; 6) porcentaje de permanencia de los estados de conducción y 7) porcentaje de permanencia promedio de los estados de conducción.

Las condiciones de marcha para los ciclos de conducción son: 1$)$ ralentí: ( $v \leq 2 \mathrm{~km} / \mathrm{h}) ; 2)$ crucero: $\left.\left(-0,1 \mathrm{~m} / \mathrm{s}^{2}<\mathrm{a}<0.1 \mathrm{~m} / \mathrm{s}^{2}\right) ; 3\right)$ aceleración: $\left(a \geq 0.1 \mathrm{~m} / \mathrm{s}^{2)}\right.$ y $(\mathrm{v}>2 \mathrm{~km} / \mathrm{h})$ y 4$)$ desaceleración: $\left(a \leq-0,1 \mathrm{~m} / \mathrm{s}^{2}\right)$ y $(\mathrm{v}>2 \mathrm{~km} / \mathrm{h})$.

La velocidad instantánea (v) en kilómetros por hora $(\mathrm{km} / \mathrm{h})$ determinó el estado en que se encuentra el vehículo durante cada segundo. A partir de la Ecuación 1 se calcula la relación distancia versus tiempo en los buses de la ciudad de Ibarra.

$v_{i}=\frac{d_{i}-d_{i-1}}{\Delta \mathrm{t}}$

Donde:

$v_{i}$ : Velocidad instantánea del bus.

$d_{i}$ : Posición instantánea del vector posición.

$\Delta \mathrm{t}$ : Variación del tiempo $(\Delta \mathrm{t}=1$ segundo $)$.

La velocidad promedio diaria de cada bus se encuentra en función de la sumatoria de la relación de la distancia que recorrieron en un determinado tiempo.

Para el cálculo de la velocidad se usa el promedio diario de la flota de autobuses de Ibarra y se utilizó la Ecuación 2, la cual se encuentra en función de la sumatoria de la velocidad promedio diaria de los buses. 
$V_{\text {PROM DIARIA FLOTA }}=\frac{1}{n} \sum_{j=1}^{n} V_{\text {PROM DIARIA BUS }}$

Donde:

$V_{\text {PROM DIARIA FLOTA }}$ V Velocidad promedio diaria de la flota.

$V_{\text {PROM DIARIA BUS }}$ : Velocidad promedio diaria del bus.

Luego, se recurre a la Ecuación 3 para obtener la velocidad promedio de las rutas totales de la semana de la flota de autobuses urbanos de Ibarra.

$\mathrm{V}_{\text {PROM SEMANAL FLOTA }}=\frac{1}{\mathrm{n}} \sum_{\mathrm{i}=1}^{7} \mathrm{~V}_{\text {PROM DIARIA FLOTA }}$

Donde:

VROM SEMANAL FLOTA: Velocidad promedio semanal de la flota.

VRROM DIARIA FLOTA: Velocidad promedio diaria de la flota.

i Día de la semana (1 a 5 = entre semana, 6 = sábado y 7 = domingo).

La velocidad instantánea $(\mathrm{m} / \mathrm{s})$ se utilizó para determinar los estados de conducción de los choferes tomados en intervalos de un segundo para disminuir el margen de error. A partir de la velocidad instantánea y la aceleración se calcularán los porcentajes de permanencia que ejecutan los conductores en los diferentes estados de marcha del vehículo. Por lo tanto, la Ecuación 4 se utiliza para el cálculo de la aceleración instantánea de los buses.

$\mathrm{a}=\frac{v_{f}-v_{o}}{\Delta \mathrm{t}}$

Donde:

a: Aceleración instantánea del bus.

$v_{f}$ : Velocidad final.

$v_{o}$ : Velocidad inicial.

$\Delta \mathrm{t}$ : Variación del tiempo $(\Delta \mathrm{t}=1$ segundo).

Luego, se realizó el cálculo de la aceleración y desaceleración promedio diaria y semanal que experimentan los buses. Los estados de conducción permitieron determinar el comportamiento o estilo de conducción que experimentan los choferes de los autobuses urbanos de lbarra. Los estados de conducción de ralentí (ER), crucero (EC), aceleración (EA) y desaceleración (ED) se calculan porcentualmente a partir de las Ecuaciones 2, 3, 4 y 5, respectivamente.

$E_{R}=\sum_{\mathrm{i}=1}^{\mathrm{n}} \frac{T_{R i}}{T_{T i}} * 100 \%$ 
Donde:

$E_{R}$ : Porcentaje de permanencia en ralentí.

$T_{R i}$ : Tiempo de permanencia en estado (R ralentí, entre otras.)

$T_{T i}$ : Tiempo total de viaje.

Estos resultados obtenidos permiten el análisis de los estados de conducción por las siguientes variables: operadora, ruta, modelo de bus, sector de la ciudad, edad del conductor y franja horaria. Por lo tanto, la Ecuación 6 sirve para la determinación de la permanencia promedio en los estados de ralentí, crucero, aceleración y desaceleración, respectivamente.

$E_{j}=\frac{1}{\mathrm{n}} \sum_{i=1} t_{j}$

Donde:

$E_{j}$ : Porcentaje de permanencia promedio en ralentí.

$t_{j}$ : Tiempo de permanencia en estado (R ralentí, etc.)

i: Día de la semana (1 a 5 = lunes a viernes; 6 = sábado y 7 = domingo).

\subsection{Elaboración y procesamiento de base de datos}

Para el procesamiento de los datos se usó el software estadístico RStudio y se tomaron las siguientes consideraciones.

Se consideraron datos inválidos al tiempo de espera que experimentan los buses, comprendido entre la finalización de una vuelta y el inicio de la siguiente. De la información procesada se obtuvo la ruta de estudio, el día en el que se realizó, el número de disco, marca, modelo, año de fabricación, edad del conductor, número de vuelta y tiempos en los diferentes estados de marcha que experimentaron los buses monitoreados.

\subsection{Obtención de resultados}

Para la obtención de resultados se aplicaron códigos de programación en RStudio y se determinaron los porcentajes en los estados de macha en ralentí, crucero, aceleración y desaceleración; así como también las velocidades y aceleraciones máximas y promedios por operadora, ruta, modelo de bus, sector, edad del conductor y horario de operación de los buses. Los Data Frames que se utilizan para el análisis de los datos son tres: Data_entre semana, Data_sábado y Data_domingo. Se realizó la sectorización de la ciudad de Ibarra mediante cuadrantes, tal como se muestra en la Figura 1. Además, se determinaron los puntos de latitud y longitud de los sectores más importantes de la ciudad, tomando en consideración variables como: sistema laboral, educativo, comercial, residencial, administrativo, social y cultural.

\section{Resultados y discusión}

Para las tablas 2, 3 y 4 se define la desviación estándar en función del nivel de confianza. Para este estudio los valores promedios fueron obtenidos con un nivel de confianza mayor o igual al $95 \%$ (p igual a 0.05). 
Figura 1. Sectorización de la ciudad de Ibarra

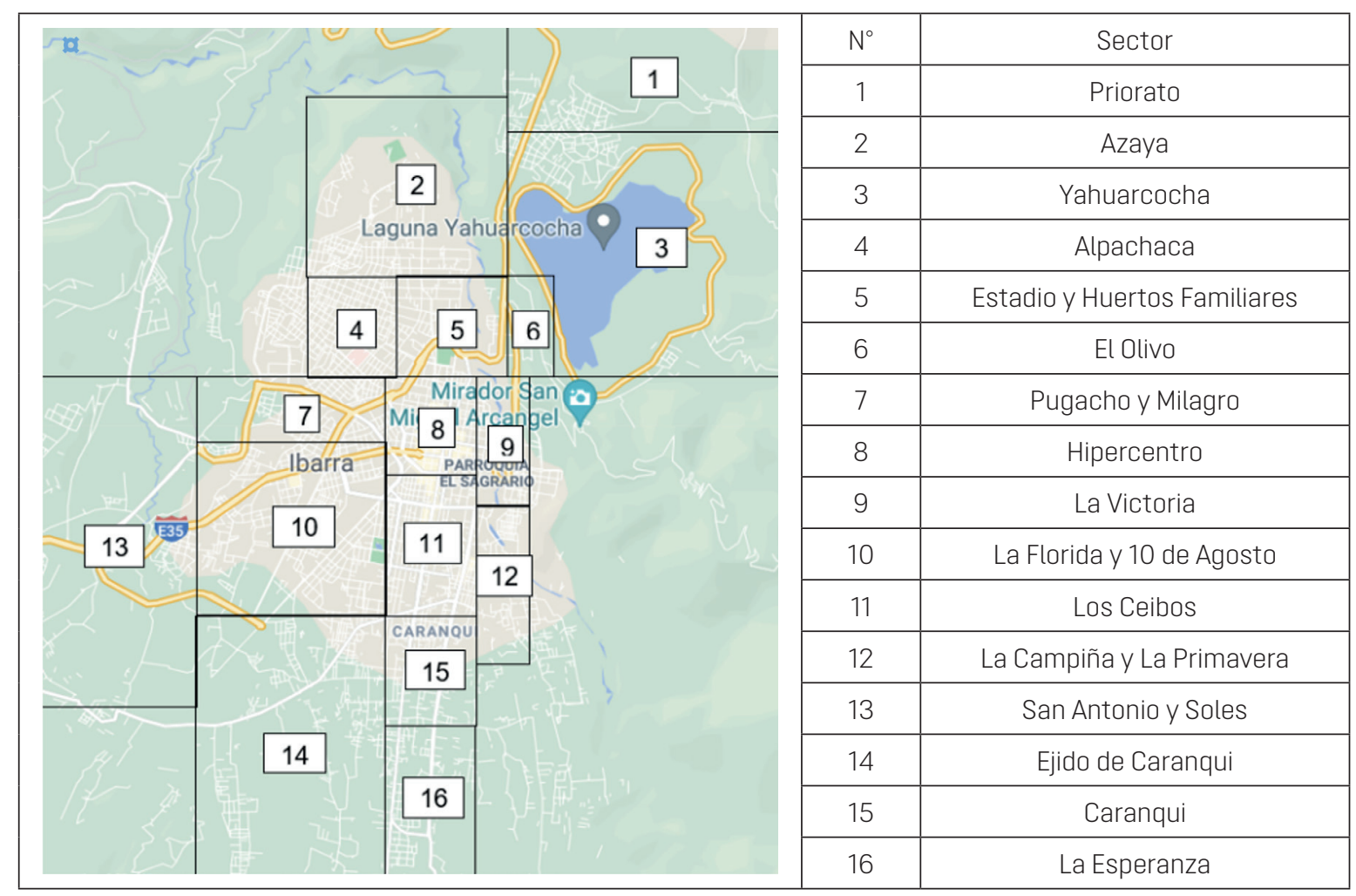

\subsection{Análisis cinemático de los estados de conducción de la flota de autobuses}

Del tiempo total de operación analizado para las 22 rutas, en la Tabla 2 se definen los estados de conducción general. En promedio, se obtuvo un 29.6\% en ralentí, un $15.2 \%$ en velocidad de crucero, el 30.9 \% en aceleración y $24.2 \%$ en desaceleración. Primero, se puede advertir que los tiempos de aceleración y desaceleración son similares con una diferencia de $6.7 \%$. Además, la velocidad de crucero del $15.2 \%$ indica que no se encuentran tramos significativos de velocidad constante dentro de la ciudad. Sin embargo, el tiempo de ralentí $29.6 \%$ es similar al tiempo de aceleración y desaceleración. Los porcentajes de permanencia de los autobuses urbanos de Ibarra no presentaron variaciones en función de la operadora, del modelo de vehículo y de la edad de los conductores, ya que los resultados muestran estilos de conducción similares.

Tabla 2. Estados de conducción general

\begin{tabular}{|l|c|c|c|c|}
\hline \multicolumn{1}{|c|}{ Días } & $\begin{array}{c}\text { Ralentí (\%) } \\
\pm \text { desviación } \\
\text { estándar }\end{array}$ & $\begin{array}{c}\text { Crucero (\%) } \\
\pm \text { desviación } \\
\text { estándar }\end{array}$ & $\begin{array}{c}\text { Aceleración (\%) } \\
\pm \text { desviación } \\
\text { estándar }\end{array}$ & $\begin{array}{c}\text { Desaceleración (\%) } \pm \\
\text { desviación estándar }\end{array}$ \\
\hline Entre semana & $29.9 \pm 2.12$ & $14.5 \pm 1.18$ & $31.3 \pm 2.17$ & $24.3 \pm 1.12$ \\
\hline Sábado & $29.2 \pm 1.55$ & $16.2 \pm 0.81$ & $30.3 \pm 1.45$ & $24.3 \pm 1.25$ \\
\hline Domingo & $28.5 \pm 0.95$ & $18.0 \pm 0.51$ & $29.7 \pm 0.85$ & $23.8 \pm 0.75$ \\
\hline Promedio & $29.6 \pm 1.54$ & $15.2 \pm 0.83$ & $30.9 \pm 1.49$ & $24.2 \pm 1.02$ \\
\hline
\end{tabular}


Los factores que tuvieron incidencia en los porcentajes de permanencia de los estados de conducción son la ruta y el sector de la ciudad.

Por ruta y en estado de ralentí, se analizó el porcentaje de permanencia en el cual los buses se encontraban en ralentí para todas las rutas durante los días entre semana, sábado y domingo, las cuales se muestran en la Figura 2. Se aprecia el estado de ralentí promedio general, el cual es de 29.6 \% y se identificó que la ruta que está más tiempo en ralentí entre semana es Católica-Alpachaca (38.3 \%). Por otra parte, la ruta que estuvo menos tiempo en ralentí fue Carmen-Bellavista (18.9\%) debido a que en esta ruta se tiene la mayor parte de su recorrido en sectores perimetrales. Para los sábados se mantuvo el mismo orden, mientras que la que menos estuvo en ralentí fue Naranjito (22.3 \%). En los domingos, la realidad fue diferente, la ruta que permaneció más tiempo en ralentí fue Católica-Alpachaca (35.2 \%) y la que estuvo en menor tiempo en ralentí fue Carmen-Bellavista (16.1\%).

Con respecto al Estado de velocidad crucero se muestra que el porcentaje de permanencia promedio general en velocidad crucero es del $15.2 \%$. Las rutas con mayor porcentaje son: Naranjito (20.6 \%) en los días entre semana, mientras que para el sábado Naranjito tuvo $(28.5 \%)$ y para el domingo la ruta Aloburo (27.9 \%).

De igual manera, las rutas que se encontraron en menor permanencia de velocidad crucero entre semana fueron: Católica-Alpachaca (11.1\%), para el sábado se mantuvo CatólicaAlpachaca (12.3 \%) y para el domingo fue 19 de Enero-Odilas (13.3\%).

Para el estado de aceleración por ruta, se ilustra el porcentaje de permanencia promedio general en aceleración que es del (30.9 \%). Las rutas en la que más se encontró el estado de aceleración son entre semana Carmen-Bellavista (33.6 \%), mientras que los sábados CaranquiAduana (32.6\%) y para los domingos Carmen-Bellavista (34.2\%).

Sin embargo, los menores índices de permanencia en estado de aceleración son: entre semana Católica-Alpachaca (27.7 \%), sábado Naranjito (25.6 \%) y domingo la ruta Santo Domingo-Universidades (29.7\%).

Se aprecia el estado de desaceleración promedio general, el cual es de (24.2\%). Se aprecia que la ruta que experimentó mayor permanencia en desaceleración en todos los días fue Carmen-Bellavista (30.1\%) entre semana, (26.5 \%) sábado y (31.5 \%) para domingo.

Mientras que la de menor índice de desaceleración fue Católica-Alpachaca (23 \%) entre semana, (21.6 \%) sábado y (21.4\%) en domingo.

Analizando los estados de conducción en base a los sectores de la ciudad: hipercentro, urbano y perimetral para los periodos entre semana, sábado y domingo, la Figura 3 muestra que entre semana (lunes a viernes) el sector en el cual los buses están más tiempo en ralentí fue en el hipercentro con el (43.4\%) de permanencia, seguido por el urbano con el $(22.4 \%)$ y, finalmente, el perimetral (21.4\%).

En el estado de aceleración la parte urbana fue el sector en el cual los buses permanecen más tiempo con un (34.3\%), seguido por la parte perimetral con un (34 \%) y, finalmente, el hipercentro con el (25.4\%). En cuanto al estado de conducción en desaceleración el orden fue similar con: (26.1\%) en la parte urbana, $(25.8 \%)$ en perimetral y $(21.2 \%)$ en hipercentro. La velocidad de crucero tuvo un (18.7\%) en el sector perimetral, (17.3\%) en el urbano y, finalmente, (10\%) en el hipercentro. 
Figura 2. Permanencia del estado por ruta (a) ralentí

(b) velocidad crucero (c) aceleración (d) desaceleración

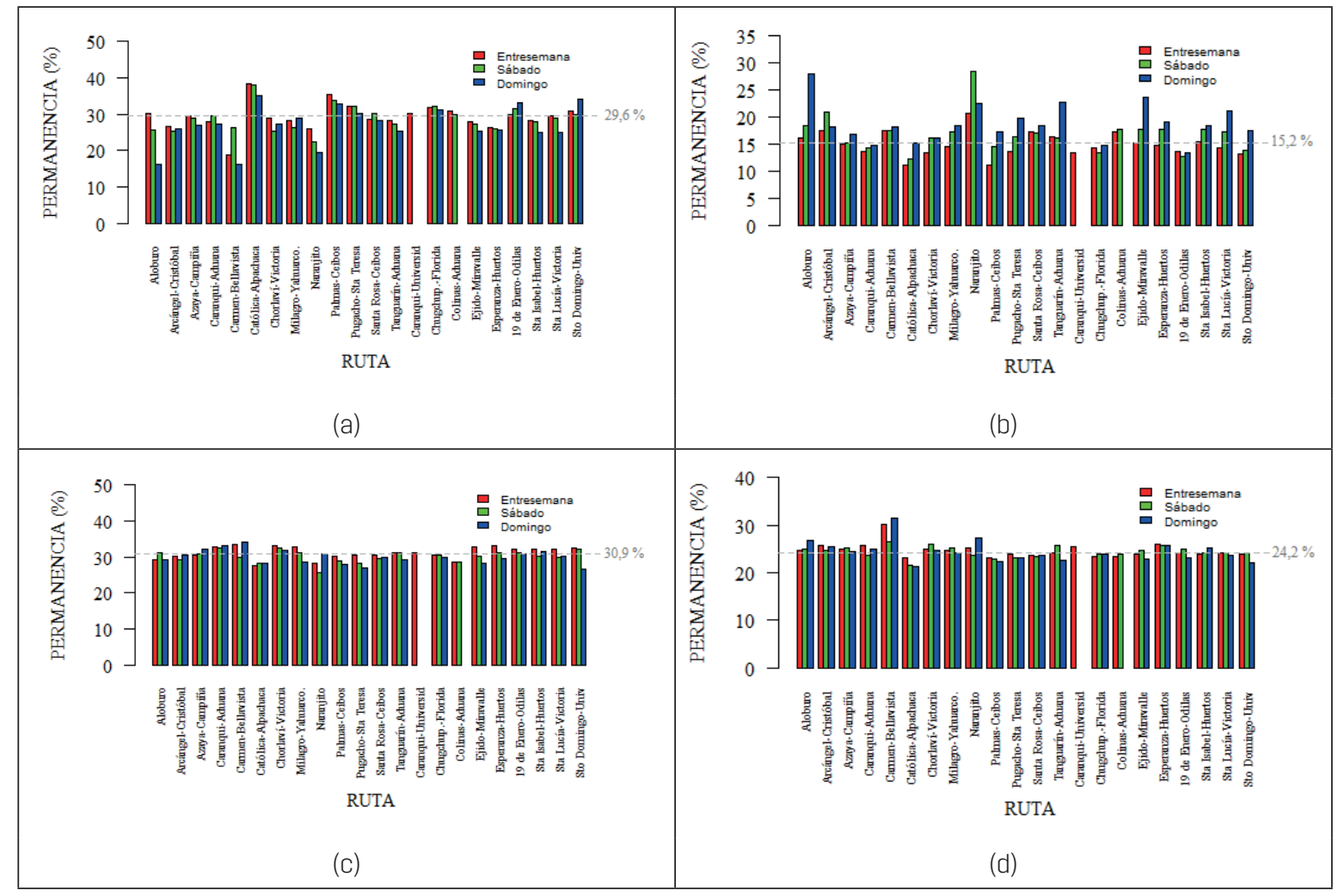

Para el estado de ralentí en los días sábados se muestra que el hipercentro tiene (44.3\%), el perimetral el (20.7\%) y, finalmente, el urbano con el (20.5 \%). El estado de aceleración en la parte urbana fue (33.7\%), seguido de la parte perimetral con un (32.8\%) y, por último, el hipercentro con el (24.2\%). El estado de desaceleración fue similar al de la aceleración con (26.5 \%) en urbano, $(25.8 \%)$ en perimetral y $(20.5 \%)$ en hipercentro. La permanencia en velocidad de crucero fue para el sector perimetral con el (20.7\%), seguido del urbano con el (19.2\%) y, finalmente, el hipercentro con el (11\%). Para los domingos, el estado de ralentí fue mayor en el hipercentro con el (44.3\%), el urbano con el (20.4\%) y, finalmente, el perimetral con el (19.5\%). El estado de aceleración en la parte del hipercentro y urbano fue (32.7\%) y, por último, el perimetral con el (23.2 \%). En cuanto al estado de conducción en desaceleración el orden fue: el perimetral con un $(25.9 \%)$ en la parte urbana $(25.7 \%)$ y $(19.9 \%)$ en el hipercentro. La velocidad de crucero tuvo un (21.9 \%) en el sector perimetral, (21.2 \%) en el urbano y, finalmente, (12.6 \%) en el hipercentro.

\subsection{Análisis cinemático de la velocidad máxima y promedio de la flota de autobuses}

En la Tabla 3 se observa que los buses alcanzan en promedio una velocidad máxima de 103.63 $\mathrm{km} / \mathrm{h}$, equivalente a $13.35 \mathrm{~km} / \mathrm{h}$ en general para los días considerados entre semana, sábado y domingo. Se pudo identificar que la mayor velocidad fue $108.5 \mathrm{~km} / \mathrm{h}$ entre semana, $96.15 \mathrm{~km} / \mathrm{h}$ para los sábados y $86.39 \mathrm{~km} / \mathrm{h}$ para los domingos. 
Figura 3. Permanencia de los estados de conducción por sector

(a) entre semana (b) sábado (c) domingo

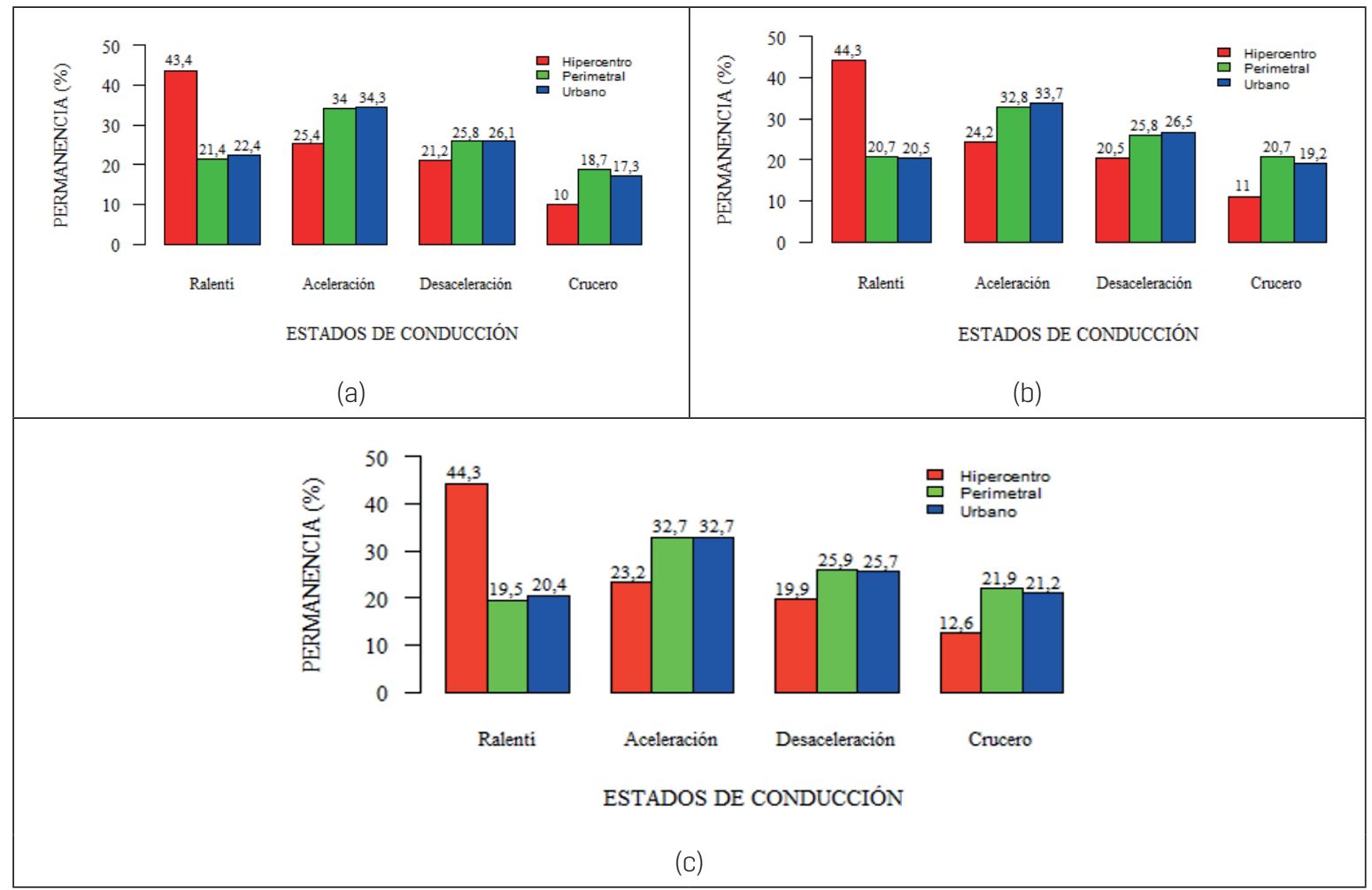

La ruta Tanguarín-Aduana fue la que presentó este mayor incremento de velocidad, en un día entre semana, a una hora pico y con un conductor de edad entre 30 y 39 años, lo cual, según el Artículo 191 del Reglamento General de Aplicación a la Ley Orgánica de Transporte Terrestre Tránsito y Seguridad Vial, superó considerablemente los límites de velocidad.

Tabla 3. Velocidad máxima y promedio general

\begin{tabular}{|l|c|c|}
\hline \multicolumn{1}{|c|}{ Días } & $\begin{array}{c}\text { Velocidad máxima }(\mathbf{k m} / \mathbf{h}) \\
\pm \text { desviación estándar }\end{array}$ & $\begin{array}{c}\text { Velocidad promedio }(\mathrm{km} / \mathrm{h}) \\
\pm \text { desviación estándar }\end{array}$ \\
\hline Entre semana & $108.583 \pm 5.51$ & $13.314 \pm 1.22$ \\
\hline Sábado & $96.156 \pm 4.82$ & $13.436 \pm 0.81$ \\
\hline Domingo & $86.396 \pm 4.32$ & $13.462 \pm 0.57$ \\
\hline Promedio & $103.638 \pm 4.87$ & $13.353 \pm 0.87$ \\
\hline
\end{tabular}

Para los resultados obtenidos no se tuvo mayor variación en función de la operadora, el día, el modelo de vehículo, la edad de los conductores y la franja horaria. Los factores que presentaron incidencia para el análisis de las velocidades fueron la ruta y el sector de la ciudad, nuevamente. Esto indica que las rutas que poseen menor velocidad promedio son aquellas que pasan por sectores urbanos e, incluso, por el hipercentro; mientras que las que adquieren mayor velocidad están conformadas, en gran parte, por tramos perimetrales.

Por ruta y con velocidad promedio se analizó la velocidad promedio para los buses que se encontraron durante los días: entre semana, sábado y domingo, la cual se muestran en la Figura 
4. Se aprecia la velocidad promedio general, la cual es de $(13.35 \mathrm{~km} / \mathrm{h})$ y se identificó que la ruta que genera mayor velocidad entre semana es la Carmen- Bellavista $(18.3 \mathrm{~km} / \mathrm{h})$. Por otra parte, la ruta que estuvo con menor velocidad fue Católica-Alpachaca $(13 \mathrm{~km} / \mathrm{h})$.

Para los sábados se mantuvo el mismo orden, Carmen- Bellavista $(17.5 \mathrm{~km} / \mathrm{h})$. Por otra parte, la ruta que estuvo con menor velocidad fue Católica-Alpachaca $(12 \mathrm{~km} / \mathrm{h})$ y, para los domingos, los resultados fueron similares, la ruta con mayor velocidad fue Carmen- Bellavista (19 $\mathrm{km} / \mathrm{h}$ ) y la de menor velocidad fue Católica-Alpachaca $(12 \mathrm{~km} / \mathrm{h})$.

Figura 4. Velocidad promedio (a) ruta (b) por sector

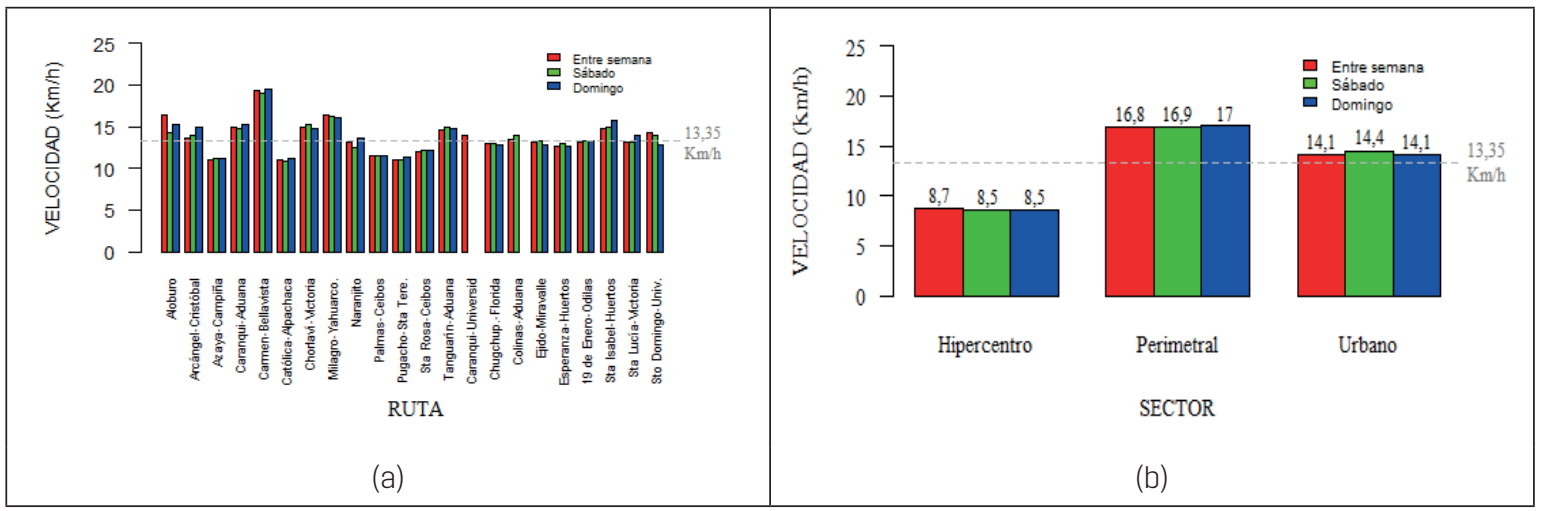

A continuación, por sector se analizaron las velocidades promedio en base a los sectores de la ciudad: hipercentro, urbano y perimetral para los periodos semana, sábado y domingo.

Se muestra que entre semana (lunes a viernes) el sector en el cual los buses presentan mayor velocidad fue la perimetral con $(16.8 \mathrm{~km} / \mathrm{h})$, en el hipercentro $(8.7 \mathrm{~km} / \mathrm{h})$, seguido por el urbano con $(14.1 \mathrm{~km} / \mathrm{h})$.

Para los días sábados, el sector que presenta mayor velocidad fue la perimetral con (16.9 $\mathrm{km} / \mathrm{h})$, en el hipercentro (8.5 km/h), seguido por el urbano con $(14.4 \mathrm{~km} / \mathrm{h})$.

Finalmente, para los domingos el sector que presenta mayor velocidad fue la perimetral con $(17 \mathrm{~km} / \mathrm{h})$, en el hipercentro $(8.5 \mathrm{~km} / \mathrm{h})$, seguido por el urbano con $(14.1 \mathrm{~km} / \mathrm{h})$.

\subsection{Análisis cinemático de la aceleración y desaceleración promedio de la flota de autobuses}

En la Tabla 4 se muestra la aceleración y desaceleración promedio general de los buses, los cuales fueron $0.158 \mathrm{~m} / \mathrm{s}^{2} \mathrm{y}-0.158 \mathrm{~m} / \mathrm{s}^{2}$, respectivamente. Entre semana la aceleración promedio es $\left(0.16 \mathrm{~m} / \mathrm{s}^{2}\right)$, para el sábado $\left(0.15 \mathrm{~m} / \mathrm{s}^{2}\right)$ y para el domingo $\left(0.14 \mathrm{~m} / \mathrm{s}^{2}\right)$.

Por otra parte, entre semana la desaceleración promedio es $\left(-0.16 \mathrm{~m} / \mathrm{s}^{2}\right)$, para el sábado $\left(-0.15 \mathrm{~m} / \mathrm{s}^{2}\right)$ y para el domingo $\left(-0.14 \mathrm{~m} / \mathrm{s}^{2}\right)$.

Por lo tanto, esto significa que el estilo de conducción en base a la aceleración y desaceleración promedio tiene un comportamiento igual.

Las mayores variaciones de aceleración y desaceleración promedio se identificaron en base a la ruta, al modelo del vehículo y al sector de la ciudad.

Por ruta, se analizaron la aceleración y desaceleración promedio para los buses que se encontraron durante los días: entre semana, sábado y domingo que se muestran en la Figura 5. Se aprecia que entre semana la aceleración promedio es de $0.15 \mathrm{~m} / \mathrm{s}^{2}$ y la desaceleración pro- 
medio es de $-0.15 \mathrm{~m} / \mathrm{s}^{2}$. Se identificó que la ruta con mayor índice de aceleración y desaceleración fue Chorlaví-La Victoria con $0.182 \mathrm{~m} / \mathrm{s}^{2}$ y $-0.182 \mathrm{~m} / \mathrm{s}^{2}$, respectivamente. Y la de menor aceleración y desaceleración promedio fue Naranjito con $0.13 \mathrm{~m} / \mathrm{s}^{2}$ y $-0.13 \mathrm{~m} / \mathrm{s}^{2}$, respectivamente.

Tabla 4. Aceleración y desaceleración promedio general

\begin{tabular}{|c|c|c|}
\hline Días & $\begin{array}{c}\text { Aceleración promedio }\left(\mathrm{m} / \mathrm{s}^{2}\right) \\
\pm \text { desviación estándar }\end{array}$ & $\begin{array}{c}\text { Desaceleración promedio }\left(\mathrm{m} / \mathbf{s}^{2}\right) \\
\pm \text { desviación estándar }\end{array}$ \\
\hline Entre semana & $0.163 \pm 0.0079$ & $-0.163 \pm 0.0079$ \\
\hline Sábado & $0.153 \pm 0.0076$ & $-0.153 \pm 0.0076$ \\
\hline Domingo & $0.141 \pm 0.0071$ & $-0.141 \pm 0.0071$ \\
\hline Promedio & $0.158 \pm 0.008$ & $-0.158 \pm 0.008$ \\
\hline
\end{tabular}

Por modelo del vehículo se analizaron la aceleración y desaceleración por modelo de vehículo y se aprecia que entre semana la aceleración promedio es de $0.15 \mathrm{~m} / \mathrm{s}^{2}$ y la desaceleración promedio es de $-0.15 \mathrm{~m} / \mathrm{s}^{2}$. Se identificó que los buses Hino GD e Hino RK alcanzan la mayor aceleración y desaceleración promedio de $0.2 \mathrm{~m} / \mathrm{s}^{2}$ y $-0.17 \mathrm{~m} / \mathrm{s}^{2}$, respectivamente, lo que significa que los buses de este tipo trabajan en perfectas condiciones.

Para los sábados se observa que los buses Hino GD e Hino RK alcanzan la mayor aceleración y desaceleración promedio de $0.17 \mathrm{~m} / \mathrm{s}^{2}$ y $-0.17 \mathrm{~m} / \mathrm{s}^{2}$, respectivamente.

Para los domingos se observa que los buses Hino FD alcanzan la mayor aceleración y desaceleración promedio de $0.15 \mathrm{~m} / \mathrm{s}^{2} \mathrm{y}-0.15 \mathrm{~m} / \mathrm{s}^{2}$, respectivamente.

Esto significa una reducción en un 13 \% en comparación a los días entre semana y en un $11 \%$ en comparación a los sábados.

A continuación, por sector se analizaron las aceleraciones y desaceleraciones promedios en base a los sectores de la ciudad: hipercentro, urbano y perimetral para los periodos semana, sábado y domingo.

Figura 5. Aceleración y desaceleración promedio por sector (a) por ruta (b) por sector

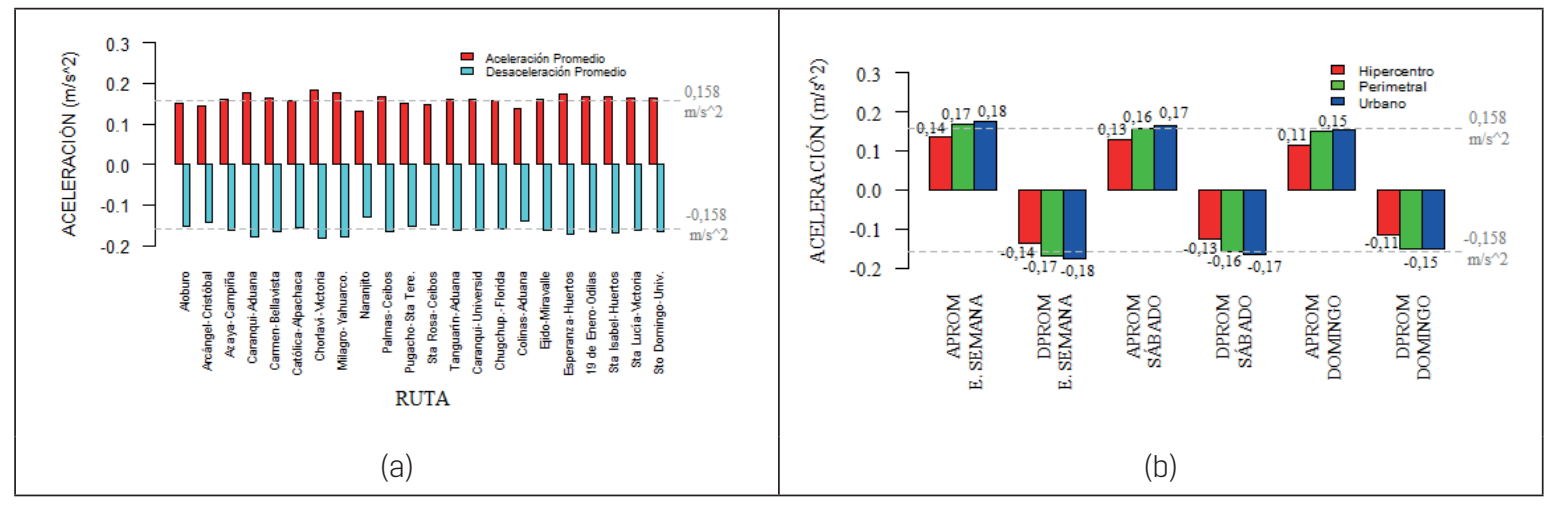

Además, se aprecia que los buses generan mayor aceleración y desaceleración promedio cuando se trasladaban por el sector urbano, seguido del sector perimetral, $y$, finalmente, el hipercentro. Entre semana, la parte urbana presentó una diferencia entre el sector perimetral e hipercentro del $4 \%$ y $22 \%$, respectivamente. Para sábados, la diferencia del sector urbano con respecto 
al perimetral e hipercentro fue del $5 \%$ y $23 \%$, respectivamente. Y, en cuanto a los domingos, el urbano tuvo una diferencia entre el sector perimetral e hipercentro en $2 \%$ y $24 \%$, respectivamente.

\section{Conclusiones y recomendaciones}

Los porcentajes de permanencia promedio de la flota de autobuses urbanos de la ciudad de Ibarra fue de 30.9 \% en aceleración; 29.6 \% en ralentí; $24.2 \%$ en desaceleración y $15.2 \%$ en velocidad crucero. Estos porcentajes de permanencia en los estados de conducción no varían en función de la operadora, del modelo de vehículo, de la edad de los conductores ni de la franja horaria que implica.

Los factores que tuvieron incidencia en los porcentajes de permanencia de los estados de conducción fueron la ruta y el sector de la ciudad. La ruta Católica-Alpachaca presentó la mayor permanencia en ralentí con 38.3 \% los días entre semana; la ruta Naranjito tuvo mayor permanencia en crucero con 28.5 \% los sábados; la ruta Carmen-Bellavista permaneció más en aceleración y desaceleración con 34.2 \% y 31.5 \% en los domingos, respectivamente. En el hipercentro fue donde se presentó más tráfico vehicular para todos los días, debido a su mayor permanencia en ralentí de $44 \%$; mientras que en el sector urbano y perimetral se obtuvieron conducciones similares.

La velocidad promedio de los autobuses fue de $13.35 \mathrm{~km} / \mathrm{h}$, los factores que presentaron incidencia fueron la ruta y el sector de la ciudad. Carmen-Bellavista fue la ruta que obtuvo mayor velocidad promedio con $19.5 \mathrm{~km} / \mathrm{h}$ y el sector perimetral presentó la mayor velocidad promedio de $16.9 \%$. La velocidad máxima registrada fue de $108.6 \mathrm{~km} / \mathrm{h}$ en un bus Chevrolet FTR de la ruta Tanguarín-Aduana en un día entre semana a una hora pico y operado por un conductor de edad entre 30 y 39 años.

La aceleración y desaceleración promedio general de la flota de autobuses fue de 0.158 $\mathrm{m} / \mathrm{s}^{2}$ y $-0.158 \mathrm{~m} / \mathrm{s}^{2}$, respectivamente. Las mayores variaciones de aceleración y desaceleración promedio se identificaron en base a la ruta, al modelo del vehículo y al sector de la ciudad. Entre semana, la ruta Chorlaví-La Victoria en el sector urbano obtuvo la mayor aceleración y desaceleración promedio de $0.18 \mathrm{~m} / \mathrm{s}^{2}$ y $-0.18 \mathrm{~m} / \mathrm{s}^{2}$, respectivamente; el Hino GD fue el modelo de bus que adquirió mayor aceleración y desaceleración promedio de $0.21 \mathrm{~m} / \mathrm{s}^{2}$ y $-0.21 \mathrm{~m} / \mathrm{s}^{2}$, respectivamente.

La información obtenida en el presente trabajo puede ser utilizada como dato de entrada para modelos de emisiones (MOOVES, PHEM, IVE, COOPERT), permitiendo la estimación de factores de emisión para vehículos en condiciones de tráfico real.

La investigación futura se centrará en examinar la variación de los patrones cinemáticos de los autobuses para diferentes niveles de tráfico vehicular. Los resultados del estudio pueden ser utilizados como línea base de políticas públicas para el desarrollo de estrategias para reducir el impacto medioambiental generado por los autobuses de transporte público en zonas urbanas.

\section{Referencias}

András, V. (2002). The Effects of Small Roundabouts on Emissions and Fuel Consumption: A Case Study. Transportation Research Part D: Transport and Environment, 1(7): 65-71. http://dx.doi. org/10.1016/S1361-9209(01)00011-6.

Cal, R., Mayor, R., y Asociados. (2005). Manual de Planeación y Diseño para la Administración del Tránsito y Transporte: Estudios de Campo Oferta y Demanda de Transporte Urbano. Bogotá, Colombia. 
D. Güler. (2016). Dynamic Analysis of a Double Wishbone Suspension (Tesis de Maestría). Izmir Institute of Technology.

Davari, M.; Jerrelind, J., y Stensson Trigell, A. (2017). Energy Efficiency Analyses of a Vehicle in Modal and Transient Driving Cycles Including Longitudinal and Vertical Dynamics. Transportation Research Part D: Transport and Environment, 53: 263-275. https://doi.org/10.1016/j.trd.2017.04.019

Ericsson, E. (2001). Independent Driving Pattern Factors and Their Influence on Fuel-Use and Exhaust Emission Factors. Transportation Research Part D: Transport and Environment, 6(5): 325-345.

Frey, C., et al. (2007). Comparing Real-World Fuel Consumption for Diesel- and Hydrogen-Fueled Transit Buses and Implication For Emissions. Transportation Research Part D: Transport and Environment, 12: 281-291.

Hernández, H. (2016). Caracterización de ciclos de conducción utilizando una tableta Android y bus CAN (Tesis de maestría). Universidad Politécnica de Cataluña

Huang, D.; Xie, H.; Ma, H., y Sun, Q. (2017). Driving Cycle Prediction Model Based on Bus Route Features. Transportation Research Part D: Transport and Environment, 54: 2007-4118. https://doi. org/10.1016/j.trd.2017.04.038

Islas, V.; Rivera, C., y Torres, G. (2002). Estudio de la demanda de transporte. Instituto Mexicano de Transporte.

Ivanic ‘, Z. (2007). Data Collection and Development of New York City Refuse Truck Duty Cycle. SAE World Conference, Detroit, USA.

Jääskeläinen, H., y Khair, M. K. (2020). Emission Standards: Europe: Heavy-Duty Truck and Bus Engines. Https://Dieselnet.Com/Standards/Eu/Hd.Php.

Jing, Z.; Wang, G.; Zhang, S., y Qiu, C. (2017). Building Tianjin Driving Cycle Based on Linear Discriminant Analysis. Transportation Research Part D: Transport and Environment, 53: 78-87. https://doi. org/10.1016/j.trd.2017.04.005

Kancharla, S., y Ramadurai, G. (2018). Incorporating Driving Cycle Based Fuel Consumption Estimation in Green Vehicle Routing Problems. Sustainable Cities and Society, 40: 214-221 https://doi. org/10.1016/j.scs.2018.04.016

Kaymaz, H.; Korkmaz, H., y Erdal, H. (2019). Development of a Driving Cycle For Istanbul Bus Rapid Transit Based on Real-World Data Using Stratified Sampling Method. Transportation Research Part D: Transport and Environment, 75: 123-135. https://doi.org/10.1016/j.trd.2019.08.023

Lee, T., y Son, J. (2011). Relationships Between Driving Style and Fuel Consumption in Highway Driving. SAE Technical Paper.

Leon, C.; Rosero, F., y Garzón, L. (2017). Consumo de combustible de las unidades de transporte urbano de la ciudad de Ibarra. Análisis comprensivo de las variables. Revista Infociencia, 1(11): 74-79.

Martínez, J. (2016). Análisis de emisiones de un vehículo en coducción por ciudad y renovación del sistema PEMS utilizado (Tesis de grado). Universidad Politécnica de Madrid.

Meixueiro, J.; Pérez, M., y Mascle, A. (2009). Guía metodológica para la evaluación de proyectos de transporte masivo urbano. Banco Nacional de Obras y Servicios Públicos.

Minetola, P.; Iuliano, L., y Calignano, F. (2015). A customer oriented methodology for reverse engineering software selection in the computer aided inspection scenario. Computers Industry. 67: 54-71.

Minett, C., et al. (2011). Eco-Routing: Comparing the Fuel Consumption of Different Routes Between an Origin and Destination Using Field Test Speed Profiles and Synthetic Speed Profiles. IEEE Society: 32-39.

Mira, J., y Arenas, B. (2018). Aplicación de modelos de redes neuronales al modelo y predicció de emisiones de autobuses urbanos (Tesis de grado). Universidad Politécnica de Madrid

Morales, J. (2014). Revista iberoamericana de producción académica y gestión educativa, vol.3, no. 6. Centro de Estudios e Investigaciones para el Desarrollo Docente, A.C (2014)

Nguyen, Y., et al. (2021). Emission Characterization and Co-Benefits of Bus Rapid Transit: A Case Study in Hanoi, Vietnam. Atmospheric Pollution Research, 12(8): 101148. https://doi.org/10.1016/j. apr.2021.101148 
Posada, J., y Gonzáles, C. (2010). Metodología para estudios de demanda de transporte público de pasajeros en zonas rurales. Revista Facultad de Ingeniería Universidad de Antioquia: 106-118.

Quilumba, J. (2015). Diseño de mapas de rutas y señalética para la Cooperativa 28 de Septiembre en la ciudad de Ibarra durante el año 2015 (Tesis de grado). Universidad Técnica del Norte.

Rohani, M. (2012). Bus Driving Behavior and Fuel Consumption. University of Southampton.

Rosero, F.; Mera, Z.; Rosero, C., y Leon, C. (2017). Análisis del consumo de combustible en autobuses urbanos por efecto de las intersecciones Semaforizadas. Caso de estudio ciudad de Ibarra. Revista de Investigación Agropecuaria y Desarrollo Sostenible, 10.

Sierra, J. C. (2016). Estimating Road Transport Fuel Consumption in Ecuador. Energy Policy, 92. 359-368. https://doi.org/10.1016/j.enpol.2016.02.008

Sigetrans. (2015). Plan Maestro de Movilidad del Cantón Ibarra. Ibarra

Silva, J.; Moura, F., Garcia, B., y Vargas, R. (2015). Influential Vectors in Fuel Consumption by an Urban Bus Operator: Bus Route, Driver Behavior or Vehicle Type?. Transportation Research Part D: Transport and Environment, 38: 94-104, http://dx.doi.org/10.1016/j.trd.2015.04.003

Wang, Z.; Chen, F.; Fujiyama, T. (2015). Carbon Emission From Urban Passenger Transportation in Beijing. Transportation Research Part D: Transport and Environment, 41: 217-227.

Yu, Q., y Li, T. (2012). Influence of Operating Characteristic of On-Road Gasoline Passenger Car on Emission in Nanjing, China. In: Proceedings of the 12th COTA International Conference of Transportation Professionals.

Yu, Q.; Li, T., y Li, H. (2016). Improving Urban Bus Emission and Fuel Consumption Modeling by Incorporating Passenger Load Factor for Real World Driving. Applied Energy, 161: 101-111. 